\title{
Commentary
}

\section{Frailty, anemia and potential future biomarkers}

\author{
Athina Kapsokoulou ${ }^{1}$ and Yannis Dionyssiotis ${ }^{2}$ \\ 'Medical School of Patras, Rio, Patras, Greece; ${ }^{2} 1^{\text {st }}$ Physical Medicine and Rehabilitation Department, National Rehabilitation \\ Center EKA-KAT, Ilion, Athens, Greece \\ All published work is licensed under Creative Common License CC BY-NC-SA 4.O (Attribution-NonCommercial-ShareAlike)
}

We would like to draw attention of our readers on Röhrig Gabrielle's article: Anemia in the frail, elderly patient ${ }^{1}$, which provides an important and under-recognized systems phenomenon.

Several studies have indicated that anemia in geriatric patients is associated with functional decline and poor outcomes, therefore anemia was identified as an additional risk factor for frailty. Anemia and elevated white blood cell count (WBC) were amongst the 13 components of the Frailty Risk Index (FRI), suggesting a possible association between anemia, frailty and inflammation ${ }^{2}$.

Indeed, it has been shown that anemia and frailty in older patients share a common pathophysiology and that the role of chronic inflammation, induced by immunosenescence associated changes and oxidative stress, is crucial in both conditions. The author refers to reviews focused on the association between inflammaging, age-associated burden of diseases and oxidative stress ${ }^{3}$ and give insight into connection between frailty and inflammaging ${ }^{4}$.

During aging the deterioration and decline of physical strength is characterized by a lifetime accumulation of intracellular and cellular damage, and trigger systemic and organic dysfunction, which is easily turned into organ failure. In the presence of individually based stress factors, this phenomenon is leading to frailty. Due to the individual heterogeneity of the aging process, there is a need of biomarkers, indicating the level of organ dysfunction and may predict morbidity, mortality, and adverse health outcome individually.

Inflammaging, as a result of the age-associated immunosenescence, contributes to organ failure at the cellular level due to the associated increase of cytokines. These cytokines might also serve as biomarkers for the advanced aging process. Cytokines are possibly released by hematopoietic stem cells. A significant association between the anemia-associated parameter red cell distribution width (RDW) and mortality is already found. RDW is rising during the last 5 years of life and elevation of RDW levels has been shown to be associated with chronic diseases and chronic inflammation ${ }^{5,6}$.

The author also pinpoints an association of oxidative stress, the so-called "oxygen-free radicals" or reactive oxygen species, and multimorbidity with eryptosis, a physiologic mechanism to remove defective erythrocytes from blood circulation, which is stimulated during inflammation ${ }^{7}$. Finally, in this article ${ }^{1}$ options for management of anemia in frail patients are provided.

To conclude we want to highlight attention to this phenomenon and, in line with the author, we propose a possible direction of future research on anemia associated parameters regarding their potential role as biomarkers in frailty.

\section{References}

1. Röhrig G. Anemia in the frail, elderly people. Clinical Interventions in Aging 2016;17:319-26.

2. Ng TP, Feng L, Nyunt MS, Larbi A, Yap KB. Frailty in older persons: multisystem risk factors and the Frailty Risk Index (FRI). J Am Med Dir Assoc 2014; 15:635-42.

3. Barnes PJ. Mechanisms of development of multimorbidity in the elderly. Eur Respir J 2015;45:790-806.

4. Wu IC, Lin CC, Hsiung CA. Emerging roles of frailty and inflammaging in risk assessment of age-related chronic diseases in older adults: the intersection between aging biology and personalized medicine. Biomedicine (Taipei) 2015;5:1.

5. Martínez-Velilla N, Cambra-Contin K, García-Baztán A, AlonsoRenedo J, Herce PA, Ibáñez-Beroiz B. Change in red blood cell distribution width during the last years of life in geriatric patients. $J$ Nutr Health Aging 2015; 19:590-4.

6. Patel KV, Semba RD, Ferrucci L, et al. Red cell distribution width and mortality in older adults: a meta-analysis. J Gerontol A Biol Sci Med Sci 2010;65:258-65.

7. Lupescu A, Bissinger R, Goebel T, et al. Enhanced suicidal erythrocyte death con-tributing to anemia in the elderly. Cell Physiol Biochem 2015;36:773-83

The authors have no conflict of interest.

Corresponding author: Yannis Dionyssiotis, $1^{\text {st }}$ Physical Medicine and Rehabilitation Department, National Rehabilitation Center EKA-KAT, Ilion, Athens, Greece

E-mail: yannis_dionyssiotis@hotmail.com

Accepted 25 February 2019 